\title{
Causes of death in Prader-Willi syndrome: Prader-Willi Syndrome Association (USA) 40-year mortality survey
}

\author{
Merlin G. Butler, MD, PhD ${ }^{1,2}$, Ann M. Manzardo, PhD¹, Janalee Heinemann, MSW³, Carolyn Loker ${ }^{3}$ \\ and James Loker, MD ${ }^{4}$
}

Background: Prader-Willi syndrome (PWS) is a rare, complex, neurodevelopmental genetic disorder that is associated with hyperphagia and morbid obesity in humans and leads to a shortened life expectancy. This report summarizes the primary causes of death and evaluates mortality trends in a large cohort of individuals with PWS.

Methods: The US Prader-Willi Syndrome Association (PWSA (USA)) syndrome-specific database of death reports was collected through a cursory bereavement program for PWSA (USA) families using a brief survey created in 1999. Causes of death were descriptively characterized and statistically examined using Cox proportional hazards.

Results: A total of 486 deaths were reported (263 males, 217 females, 6 unknown) between 1973 and 2015, with mean age of $29.5 \pm 16$ years (2 months-67 years); $70 \%$ occurred in adulthood. Respiratory failure was the most common cause, accounting for $31 \%$ of all deaths. Males were at increased risk for presumed hyperphagia-related accidents/ injuries and cardiopulmonary factors compared to females. PWS maternal disomy 15 genetic subtype showed an increased risk of death from cardiopulmonary factors compared to the deletion subtype.

Conclusions: These findings highlight the heightened vulnerability to obesity and hyperphagia-related mortality in PWS. Future research is needed to address critical vulnerabilities such as gender and genetic subtype in the cause of death in PWS.

Genet Med advance online publication 17 November 2016

Key Words: cardiac and respiratory failure; GI problems; genetic subtypes; obesity; thromboembolism

\section{INTRODUCTION}

Prader-Willi syndrome (PWS) is a rare, complex, neurodevelopmental genetic disorder with multiple cognitive, behavioral, and endocrine abnormalities and recognizable physical changes. The disorder is characterized by a narrow bifrontal diameter, almond-shaped eyes, short upturned nose, and downturned corners of a dry mouth, with sticky saliva, enamel hypoplasia, and dental caries. Severe central hypotonia, poor sucking, and feeding difficulties are cardinal features during infancy. Hypogonadism and hypogenitalism have been noted in both males and females, with cryptorchidism and a micropenis in males. Growth hormone deficiency leads to short stature and small hands with a flattened ulnar border and small feet. If uncontrolled, hyperphagia (the unrelenting pathologic urge to consume food and unremitting hunger) can lead to dangerous food-seeking behavior with life-threatening obesity. ${ }^{1-5}$ Decreased muscle mass and increased fat mass are accompanied by a low metabolic rate-approximately $60 \%$ of normal. ${ }^{1,2,6}$ Limited treatment options are available for the intractable obesity and hyperphagia, which continues throughout adulthood and diminishes the quality of life for those with this disorder as well as their family members. Delayed developmental milestones and mental deficiency (average IQ = 65) for the family background are typical, and behavioral problems-including tantrums, stubbornness, obsessive compulsions and skin picking-frequently occur in childhood and continue into adolescence and adulthood.$^{1-3,7}$

PWS is the most common known cause of morbid obesity in humans, with a prevalence of 1 in 10,000-30,000 live births. ${ }^{1-3,7,8}$ The annual mortality rate is estimated at $1-4 \%$; the shortened life expectancy is greater than anticipated by patients' level of intellectual disability and is due primarily to complications of hyperphagia and obesity-related causes. ${ }^{8}$ The role of obesity as a cause of death in PWS is often discussed as a consequence of cardiorespiratory failure. ${ }^{9}$ The characteristic food-seeking behavior increases the risk for mortality because of the potential for gastrointestinal perforation, aspiration/ choking due to rapid consumption, and hypotonia-related swallowing difficulties. Accidental deaths (e.g., traffic fatalities, hypothermia) and other physiological and genetic differences unique to this syndrome further increase the mortality rate of PWS. ${ }^{2}$

PWS is caused by errors in genomic imprinting from lost expression of paternal genes in the chromosome 15q11-q13 region, most commonly due to a de novo deletion (approximately $70 \%$ of cases) followed by maternal disomy 15 (UPD15)

${ }^{1}$ Department of Psychiatry and Behavioral Sciences, University of Kansas Medical Center, Kansas City, Kansas, USA; ${ }^{2}$ Department of Pediatrics, University of Kansas Medical Center, Kansas City, Kansas, USA; ${ }^{3}$ Prader-Willi Syndrome Association (USA), Sarasota, Florida, USA; ${ }^{4}$ Bronson Children's Hospital, Kalamazoo, Michigan USA.

Correspondence: Merlin G. Butler (mbutler4@kumc.edu) 
or both 15 s from the mother (approximately $25 \%$ of cases), with the remaining cases having imprinting defects..$^{2-5}$ Imprinting defects may be inherited, with the potential of subsequent children having a PWS risk of 50\%. The PWS genetic subtypes include the most frequent paternally derived 15q11-q13 deletion consisting of the larger typical type I deletion or the smaller type II deletion involving two different proximal 15q11-q13 breakpoints or, rarely, atypically sized cytogenetic deletions. ${ }^{2,5,10-12}$ Clinical and behavioral differences have been reported in those with the two typical type I and type II deletions and those with maternal disomy 15 . Generally, those with PWS and the larger type I deletion have more behavioral problems, obsessions, and self-injury than those with the smaller type II deletion or maternal disomy $15^{2,4,13-15}$ Reduced pain threshold further increases propensity toward self-injury and infection, possibly related to disturbances in beta endorphin and substance P levels. ${ }^{16}$ Differential behavioral and physiological profiles and vulnerabilities may also be associated with maternal disomy 15 due to enhanced genetic activation with two maternally expressed alleles in the imprinted region or possible expression of recessive alleles.

Several clinical features of PWS suggest a role for hypothalamic dysfunction, including eating disturbances (hyperphagia), growth hormone deficiency, and small hands and feet, along with other endocrine problems, such as hypogenitalism and infertility. ${ }^{1,2,17}$ These and other medical findings in PWS such as dysregulation of temperature, ${ }^{18}$ particularly in infants and young children may mask signs of infectious diseases and septic shock soon after presentation..$^{19,20}$ Infants with PWS who are hospitalized may also be at risk for complications related to medical care and treatment because of a narrow upper airway, an increased likelihood of aspiration, central hypotonia, hypoventilation, swallowing difficulties, or gastroenteritis symptoms.

Herein, we report our experience in the largest study to date regarding the causes of death in PWS, utilizing the US PraderWilli Syndrome Association (PWSA (USA)) syndrome-specific mortality survey database spanning the past 40 years.

\section{MATERIALS AND METHODS}

PWSA (USA) is a nonprofit parent support organization founded by PWS families to supply information and assist or support families and others caring for those affected with this disorder. It offers a program for PWS families in which supportive bereavement information is provided to those who contact the organization in the first or second year following a death. Data regarding causes of death have been collected through this cursory program using a brief survey created in 1999, and reported deaths have consistently been tracked through the bereavement coordinator since 2001. A collaborative group of PWS experts was created in 2005 in order to develop a detailed questionnaire that contained research questions for families and requested the release of medical records, including relevant information regarding demographics, medical history, cause of death, autopsy reports, and the circumstances around the time of death. The questionnaires were disseminated to families known to have experienced the death of a relative with PWS. The collected data were reviewed by the same PWS experts. Inconsistencies in the data were reviewed and results clarified and confirmed by consulting with the reporting family member. The reported causes and contributors to each death and autopsy reports were evaluated by a clinically licensed cardiologist, parent, and expert in the care of PWS, who assessed the primary cause of death for each individual. The defined causes of death were then classified into major categories.

\section{Statistical analysis}

Causes of death for PWS fell into 13 categories that were descriptively characterized (e.g., frequencies and means with standard deviations) by gender, age, and body mass index (BMI) at death and PWS genetic subtype. The frequencies of the 13 causes were summarized and reported for four age groups: infants (newborn to $<3$ years), children ( 3 to $<12$ years), adolescents ( 12 to $<18$ years), and adults ( $\geq 18$ years). After the data set had been reviewed, causes of death were further subclassified as (i) cardiopulmonary in origin, which included all deaths due to cardiovascular or respiratory causes and pulmonary embolisms, or (ii) presumed causes considered hyperphagic in origin, including gastrointestinal problems, choking, and accidents likely to have resulted from food-seeking behaviors (e.g., crossing a street carelessly to gain access to a restaurant). Mortality trends in the age of death were also considered over time based on the year the death occurred in order to assess cohort effects related to changes in diagnostic capabilities and treatment.

Descriptive characterization, including frequencies and means with standard deviations, and all statistical comparisons were performed using SAS Statistical Software version 9.4 (Cary, NC). Pearson correlation and analysis of variance were utilized for continuous variables; bivariate and multivariate analyses of dichotomous, ordinal data used chi-square and logistic regression modeling. Cox proportional hazards ratios and log-rank testing were applied to model mortality risk as a function of the age at death and for the generation of Kaplan-Meier curves to test the effects of gender (male versus female), cause of death (cardiopulmonary versus hyperphagia), and PWS genetic subtype (deletion or maternal disomy 15) on mortality risk.

\section{RESULTS}

Our investigations compiled family-reported deaths occurring between 1973 and 2015 for 486 individuals with PWS ( $n=$ 263 (54\%) male; $n=217$ (45\%) female; $n=6$ (1\%) unknown; Table 1). The age at death was noted for 425 subjects, with an average of $29.5 \pm 16$ years, and ranged from 2 months to 67 years; it was significantly lower among males (28 \pm 16 years) than among females ( $32 \pm 15$ years) $(\mathrm{F}=6.5 ; P<0.01)$. The majority of reported deaths occurred in adulthood $(n=338 ; 70 \%)$, with only $20 \%$ of deaths observed among individuals younger than 18 years. This relationship was true even after consideration of cohort effects and changes in reporting/diagnostic capabilities with time. The distribution of adult deaths was evenly divided 
Table 1 PWSA(USA) 40-year mortality survey syndrome-specific database summary

\begin{tabular}{|c|c|}
\hline Characteristic, total $N=486$ & Value \\
\hline \multirow[t]{2}{*}{ Gender, $N=480(99 \%)$} & $N=263(54 \%)$ Male \\
\hline & $N=217(45 \%)$ Female \\
\hline Age of death, $N=425(87 \%)$ & Mean $( \pm S D)=29.5( \pm 15)$ years (range 2 months -67 years $)$ \\
\hline Male, $N=224(53 \%)$ & Mean $( \pm S D)=27.8( \pm 16)$ years (range 1 months -61 years $)$ \\
\hline Female, $N=199(47 \%)$ & Mean $( \pm S D)=31.7( \pm 15)$ years (range 2 months -67 years $)(F=6.5 P<0.011$ (gender) \\
\hline \multirow[t]{5}{*}{ Age range } & Infant (<3 years), $N=21(4 \%) ; N=13(62 \%)$ Male \\
\hline & Child ( $3-12$ years), $N=43(9 \%), N=28(65 \%)$ Male \\
\hline & Adolescent (12-18), $N=23(5 \%), N=15(65 \%)$ Male \\
\hline & Adult (18+ years), $N=338(70 \%), N=168(50 \%)$ Male \\
\hline & Undefined, $N=61(12 \%)$ \\
\hline Year of death & $N=440(90 \%) ;$ See Figure 1 \\
\hline \multirow[t]{2}{*}{ Cause of death } & Known cause, $N=312(64 \%), 13$ categories (see Figure 2 ) \\
\hline & Unknown cause, $N=174(36 \%)$ \\
\hline \multirow[t]{5}{*}{ PWS subtype, $N=48$ (10\%) } & Deletion, $N=31$ \\
\hline & Uniparental maternal disomy $15, N=14$ \\
\hline & Imprinting defect, $N=1$ \\
\hline & Acquired through injury/surgery, $N=2$ \\
\hline & Unknown genetic subtype, $N=438$ \\
\hline Autopsy performed & $N=40(8 \%)$ \\
\hline \multirow[t]{2}{*}{ Body mass index $\left(\mathrm{kg} / \mathrm{m}^{2}\right), N=132$} & Mean $( \pm S D)=49.3 \pm 23($ range $14-122) \mathrm{kg} / \mathrm{m}^{2}$ \\
\hline & Mean $( \pm S D)=92 \pm 16($ range $3-99)$ percentile \\
\hline
\end{tabular}

PWS, Prader-Willi syndrome.

(50/50) between males and females, but male gender predominated significantly among the younger age groups (64/36 male/ female; $\chi^{2}=5.7 ; P<0.02$ ).

The year of death was available for $440(90 \%)$ respondents ( $n=203$ females; $n=234$ males; $n=3$ unknown); mean age of death was reported for 425 (87\%) respondents $(n=199$ females; $n=224$ males; $n=2$ unknown) and a more precise cause of death was provided for $312(64 \%)$ individuals ( $n=137$ females; $n=174$ males; $n=1$ unknown) reported to have PWS. PWS genetic subtype data were available for $10 \%$ of individuals and were composed primarily of the deletion subtype. The mean age of mortality was positively correlated with the year of death $(r=0.12 ; P<0.01)$ in the overall sample, suggesting increased life span possibly related to improvement in medical care with time and earlier diagnosis; however, this relationship was driven by an effect in females (year of death: $r=0.20 ; P<0.005$ ). No significance was noted when only males were considered (year of death: $r=0.05 ; P=0.49$; see Figure 1).

\section{Causes of death}

A precise cause of death was available for 312 of the 486 patients and divided into 13 categories (see Figure 2). Those with and without a known cause of death did not significantly differ by gender or age at death. Considering only those with a known cause of death, the most common single cause of death was respiratory failure, reported for 98 individuals (31\%), followed by cardiac disease/failure $(n=51 ; 16 \%)$, gastrointestinal (GI) problems such as perforation, distension, or obstruction $(n=$ $31 ; 10 \%)$, and infections $(n=29 ; 9 \%)$. Obesity was listed as a cause of death for 22 patients (7\%) and pulmonary embolism for 21 patients (7\%). Additional categories reflected diseaseassociated behavioral risks associated with choking, accidents, and hypothermia, possibly related to food seeking and consumption. There were no significant differences in the frequency of male versus female gender for any individual cause of death.

Causes of death for PWS varied according to age range, with reported respiratory failure being the leading cause of death for all groups; presumed obesity-related cardiopulmonary factors contributed to more than half of all deaths (see Supplementary Figure S1 online). Deaths due to obesity-related factors such as cardiovascular disease and failure, pulmonary embolism, and renal failure appeared in childhood and increased in adolescence through adulthood, whereas deaths due to GI problems and infections were stable, at approximately $10 \%$, through the life span. Additional morbidity possibly related to food seeking (e.g., accidents, choking) appeared in childhood and adolescence and contributed to approximately one-third of all deaths and approximately half of the deaths (predominantly in males) of those younger than 18 years. BMI at the time of death was identified for 132 patients, with an average of $49 \pm 23 \mathrm{~kg} / \mathrm{m}^{2}$ and range of 14-122. Standardized mean BMI values were well above normal (>90th percentile).

\section{PWS genetic subtype}

Investigation of the relationship between PWS genetic subtype and cause of death was hindered by a lack of necessary genetic characterization in the selected sample, particularly for the older individuals who may not have had access to accurate 


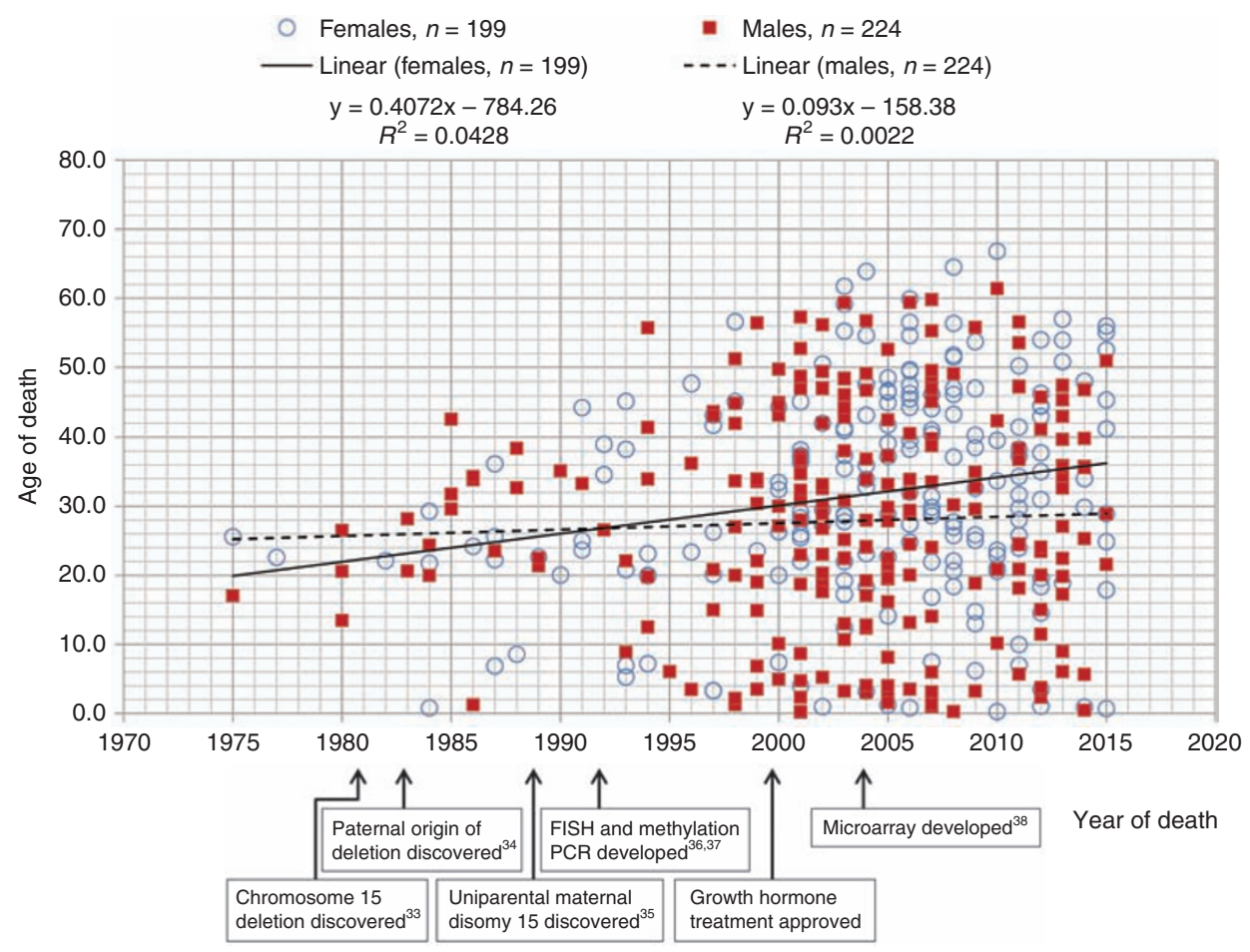

Figure 1 Relationship between the reported age and year of death for Prader-Willi syndrome. Scatter plot of the age at death by the year of death for males (red squares) and females (blue circles) with Prader-Willi syndrome (PWS) with respect to historical benchmarks in genomic discovery and treatment advances in PWS. Trend lines show the correlation coefficient for females (solid line) and males (dotted line).

genetic testing. DNA methylation testing, which is $99 \%$ accurate in confirming the diagnosis of PWS but does not determine the specific genetic subtype (chromosome 15q11-q13 deletion, maternal disomy 15 , or imprinting defects), did not become readily available until the mid- to late 1990 s. $^{21,22}$ The deletion subtype was approximately equally divided among male $(n=14 ; 45 \%)$ and female $(n=17 ; 55 \%)$ patients, with a mean age of $34.8 \pm 16$ years at death, which was not related to the year in which the death occurred. However, the age of death for individuals with the deletion subtype was significantly higher among females $(41.0 \pm 13.3$ years, range 14.755.3 years) than among males ( $27.2 \pm 16$ years, range $0.97-59$ years; $t=2.0 ; P<0.05)$. The leading causes of death associated with the deletion subtype were cardiac problems $(n=9 ; 30 \%)$, respiratory failure $(n=8 ; 27 \%)$ and infections $(n=5 ; 17 \%)$, followed by GI problems $(n=4 ; 13 \%)$, pulmonary embolism ( $n=2 ; 7 \%)$, choking $(n=1 ; 3 \%)$, and hypothermia $(n=1 ; 3 \%)$; females comprised seven of the nine cases of cardiac problems and five of eight cases of respiratory failure in the deletion subsample. The limited data from uniparental maternal disomy 15 showed that 8 of the 12 patients ( 9 males; 3 females) died from respiratory failure and the remaining 4 patients ( 3 males; 1 female) died from GI problems, cancer, infections, and renal complications, respectively, at a mean age of $22.2 \pm 18$ years (range 1.2 to 49 years), which did not differ by gender. A single male with an imprinting defect died from GI perforation at 13 years of age.

\section{Survival analyses}

Cox regression analysis of our sample of 425 individuals with PWS and a known age at death identified quartile point estimates of $25 \%$ mortality for those 20 years of age (95\% CI 18-21 years); $50 \%$ mortality for those 29 years of age (95\% CI $27-32$ years); and $75 \%$ mortality for those 42 years of age (95\% CI $39-$ 44 years). A $99 \%$ mortality rate was achieved for those 60 years of age. A primary sex difference in mortality risk was identified for PWS, with males displaying a significantly higher risk of early mortality compared with females $\left(\chi^{2}=5.0 ; P<0.025\right.$; hazard ratio $=1.2$; 95\% CI 1.0-1.5; Figure 3a). Furthermore, sex significantly interacted with the primary cause of death (cardiopulmonary versus hyperphagic), with males showing a significantly higher risk of death due to presumed hyperphagia-related causes (accidents, choking, GI problems) relative to females (Figure 3b; Supplementary Table S1 online). There was a nearly significant trend toward increased risk for cardiopulmonary-related versus hyperphagia-related deaths among females. PWS genetic subtype also significantly impacted mortality risk over the life span, with individuals possessing the maternal disomy 15 subtype showing a significantly higher risk $\left(\mathrm{HR}=2.0 ; 95 \%\right.$ CI $\left.1.0-3.9 ; \chi^{2}=4.1 ; P<0.04\right)$ of death due 


\begin{tabular}{|ll|}
\hline Category & Mean age (SD) \\
\hline Respiratory failure, $n=94$ & $24.6(16)$ years \\
\hline Cardiac, $n=50$ & $32.1(14)$ years \\
\hline GI, $n=30$ & $32.4(16)$ years \\
\hline Infection, $n=29$ & $35.7(16)$ years \\
\hline Obesity, $n=22$ & $30.7(12)$ years \\
\hline Pulmonary embolism, $n=19$ & $34.1(12)$ years \\
\hline Choking, $n=18$ & $30.1(17)$ years \\
\hline Accident, $n=17$ & $25.0(16)$ years \\
\hline Renal failure, $n=7$ & $34.2(11)$ years \\
\hline Neurologic, $n=6$ & $18.0(21)$ years \\
\hline Hypothermia, $n=3$ & $30.8(14)$ years \\
\hline Drug reaction, $n=3$ & $25.1(9)$ years \\
\hline Cancer, $n=4$ & $39.7(27)$ years \\
\hline
\end{tabular}

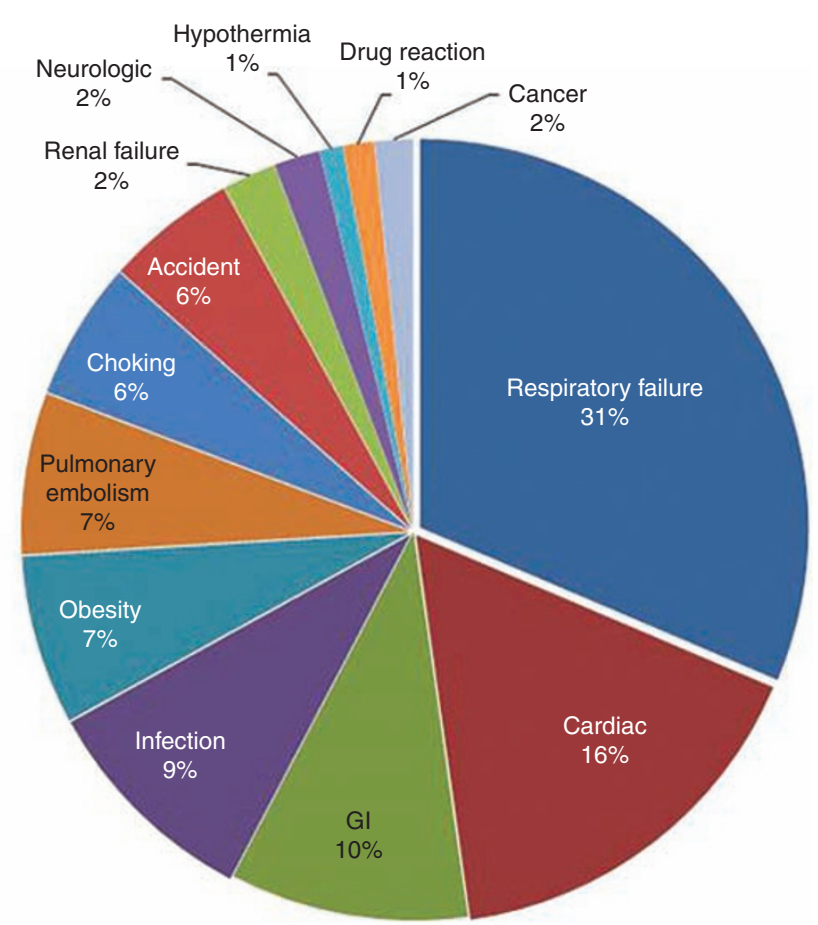

Figure 2 Causes of death among 312 individuals with Prader-Willi syndrome divided into 13 major categories.

to cardiopulmonary versus hyperphagic causes compared with the deletion subtype (Figure 4).

\section{DISCUSSION}

We report a descriptive analysis of mortality data collected from individuals with PWS with reported deaths between 1973 and 2015 as part of a supportive bereavement program for PWS families. Respiratory failure was the most common overall cause of death reported in our database of adults and children with PWS, whereas cardiac disease and failure with pulmonary thromboembolism were more commonly found in adulthood in combination with obesity-related morbidity. This is the first report to characterize and quantify deaths attributable to pulmonary embolism in PWS; it accounted for $7 \%$ of all deaths. Cardiopulmonary and BMI-related mortality factors predominated among females, whereas males were more likely to experience accidents, choking, and infection at a young age. Accidents, aspiration, sepsis, and choking were the most common causes of death in children and adolescents, accounting for approximately half of all deaths in childhood and one-third of adult deaths. A progressive increase in the life span (measured as age at mortality) was observed over time, particularly for deaths due to cardiac problems in females, possibly due to protective effects associated with earlier diagnosis, treatment intervention and monitoring (e.g., growth hormones), and weight management of recent cohorts. This was not observed among males, possibly owing to an increased rate of hyperphagia-related rather than obesity-driven cardiopulmonary deaths among males with PWS. A significantly increased risk of mortality due to cardiopulmonary factors was observed for maternal disomy 15 compared with deletion genetic subtypes, which may reflect differential vulnerability due to amplified effects of homozygous mutations from duplicated maternal contributions on chromosome 15.

Despite the report of causes of death in PWS in individual case reports, case series, population surveys, and syndromespecific registry databases for both children and adults, there is a paucity of data for systematic collection and analysis of cause of death. For example, the Cambridge PWS study in 2004 reported on fewer than 100 individuals with confirmed PWS and found a mortality rate of $3 \%$ per year after the age of 5 years. More recently, in 2012, Lionti et al. ${ }^{23}$ reviewed an Australian registry of 163 individuals with PWS from ages 3 weeks to 60 years; 15 deaths were recorded, corresponding to an $87 \%$ probability of survival to 35 years of age, which equates to a survival rate reported by an Italian survey of $80 \%$ at 40 years of age for 425 individuals with PWS. The most common causes of death were the following, in no particular order: pulmonary thromboembolism, sepsis, accidents, diabetes, cardiac disease and problems, choking, aspiration, gastric rupture, respiratory failure, and obesity-related complications. ${ }^{23-30}$

The present summary of mortality in PWS is consistent with earlier reports indicating the young age of death and high rates of mortality due to cardiac and respiratory failure. Deaths attributable to obesity-related and cardiac diseases can be anticipated for this highly vulnerable group. Our data suggest that early interventions have impacted disease trajectory, resulting in delayed mortality (i.e., older age of death) among females. However, the underlying pathology for respiratory failure-the leading cause of death for adults and children-remains elusive 


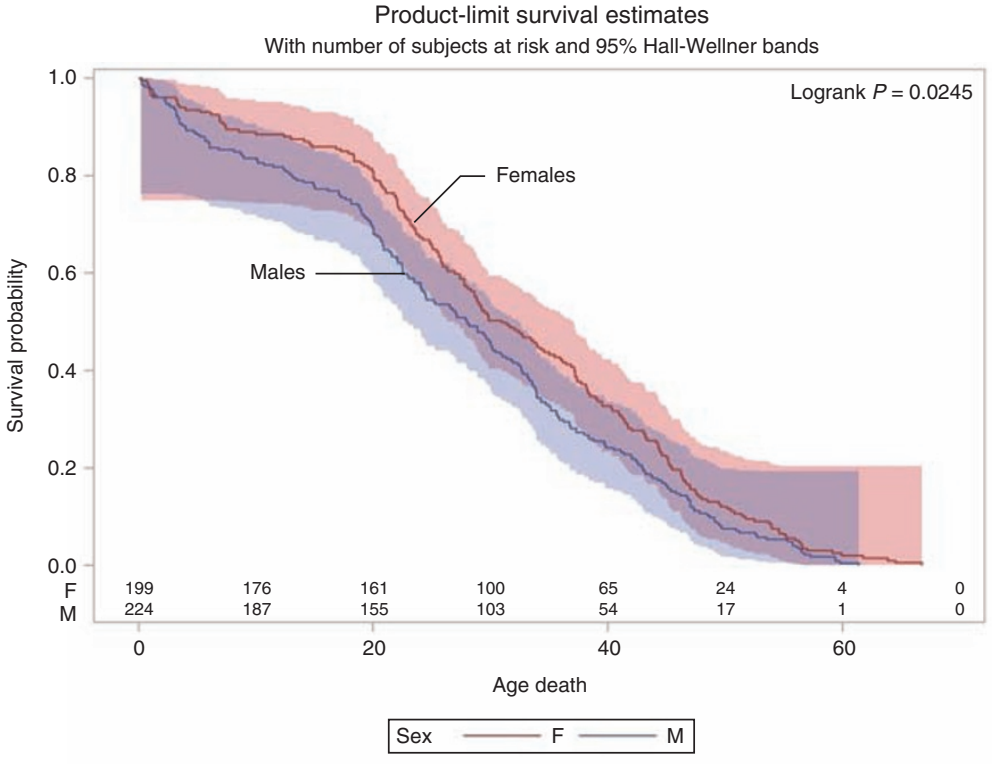

b

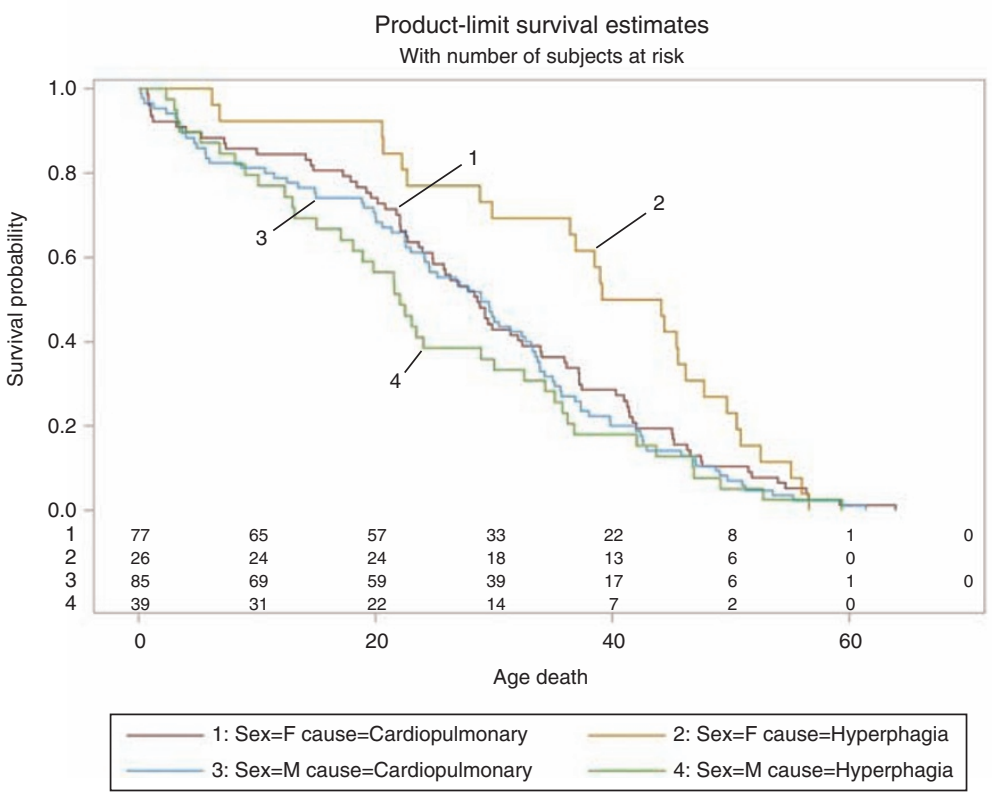

Figure 3 Survival analysis illustrates the effects of gender and cause of death on age of death for Prader-Willi syndrome. Kaplan-Meir plot of survival probability is shown as a function of age at death for a) males versus females with $95 \%$ Hall-Wellner bands and for b) cardiopulmonary versus hyperphagia-related causes by gender. Numbers of uncensored participants by group are listed at the bottom of each figure.

and does not appear to be impacted by recent advancements in treatment modalities. Reported deaths due to respiratory failure could also be secondary to undiagnosed aspiration, pulmonary embolism, or some unspecified neurological disturbance and may vary by gender and/or age. Future targeted research should seek to characterize this phenomenon to advance treatment development. Similarly, individuals with PWS have high rates of choking, accidents, and GI perforation, presumably related to uncontrolled hyperphagia and food-seeking behaviors, contributing to approximately one-third of all reported deaths and approximately half of all deaths in childhood. Accidental and/ or hyperphagia-related deaths appear to disproportionately impact younger males, possibly owing to increased activity and/or impulsive characteristics.

Early diagnosis of PWS and prevention of overweight are key factors in preventing early causes of death in individuals. These include close monitoring and supervision of food access and quantity to avoid choking caused by eating quickly and gastric rupture resulting from excessive food consumption The risks associated with choking are greater in the PWS population, ${ }^{27}$ with potential causes of increased choking related to poor oral/ motor coordination, poor sensation, hypotonia, hyperphagia, 


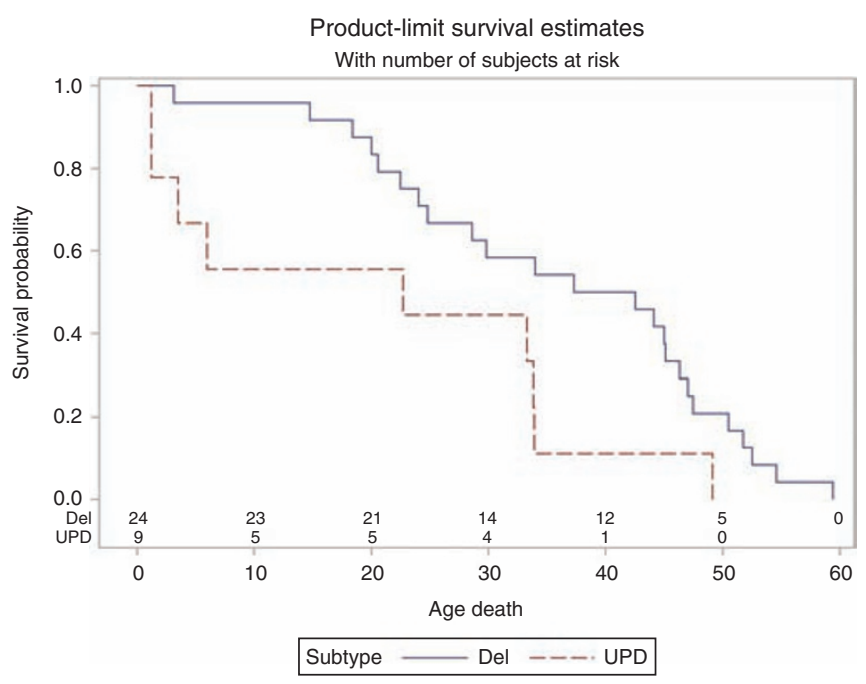

Figure 4 Survival analysis of deaths due to cardiopulmonary causes as a function of Prader-Willi syndrome (PWS) genetic subtype. Kaplan-Meir plot of survival probability is shown for deaths attributable to cardiopulmonary versus hyperphagia-related causes for individuals with 15q11-q13 deletion (Del) versus maternal uniparental disomy 15 (UPD). Numbers of uncensored participants by PWS genetic subtype are listed at the bottom of the figure.

a dry mouth, decreased mastication, and voracious feeding habits. Therefore, implementation of preventive measures and education, with better awareness of group home-care providers, are recommended, including training of the Heimlich maneuver, supervised meals, food security, food preparation, and diet modification. One operational strategy is sipping fluid between bites of food to help clear the esophagus to lessen the risk of choking. Pain in the upper abdomen and/or vomiting should be taken as a possible sign of an emergent event such as acute serious gastric/intestinal dilatation with risk for rupture. An established algorithm for evaluating individuals with PWS and GI symptoms (available at http://www.pwsausa.org) should be followed, along with intravenous fluid support.

In adults, weight reduction is complicated by daytime sleepiness due to apnea. Adults with PWS and obesity can be expected to have the same life-threatening complications as obese individuals without PWS, including complications of cardiopulmonary problems, hypertension, diabetes, and skin infections. ${ }^{31}$ Weight reduction lowers the risk of obesity-related, possibly life-threatening complications.

Because hyperphagia and subsequent obesity are cardinal features of this rare genetic disorder, excessive overeating, stomach necrosis and rupture, cardiovascular disease, respiratory failure, sleep apnea, diabetes mellitus, and related comorbidities can be life-threatening. Early diagnosis, dietary intervention with restricted caloric intake, and significant controls of access to food (e.g., locking cabinets) along with exercise programs developed for those with PWS have led to successful weight loss and BMI reduction; active management of hyperphagia prolongs life. Furthermore, the use of growth hormones and other hormone replacements have helped to normalize body composition and stature in PWS, with a positive impact on the control of obesity.

In summary, causes of death seen in infancy or young childhood in PWS are more likely to be related to respiratory failure, aspiration, infection, and choking than to obesity-related factors, whereas cardiac disease and failure, pulmonary thromboembolism, accidents, sepsis, and obesity-related complications are more commonly found in adolescents and adulthood. Deaths from cardiac disease typically reflect right heart failure rather than atherosclerotic disease. Males may be more likely to engage in aggressive or risky food-seeking behaviors than females, particularly in childhood, whereas females may be more likely to experience obesity-related morbidity. Standardized syndrome-specific growth charts developed for growth hormone-treated individuals with PWS appear to show differences in the degree of obesity (weight) between males and females. At age 18 years, females with PWS were heavier than males of the same age with PWS. ${ }^{32}$

Our analyses and interpretation of data trends are limited by the reliability of death reporting based on the availability and knowledge of family members and access to confirmed genetic status and autopsy reports. Older individuals without living relatives to report the death may have been underreported. Similarly, deaths of infants and children with PWS, prior to better awareness with recognition of PWS and the availability of advanced genetic testing, may have escaped diagnosis prior to death. Statistical corrections for age and time effects have been incorporated to minimize the influences of these factors on our conclusions, but the sample size is small. Gender differences in PWS genetic subtype may have influenced the result. Nevertheless, this study is the largest and most extensive examination of mortality due to PWS to date. The results support the current understanding of disease pathology and mortality for PWS and provide useful insight into risk factors, mortality trajectory over time, and areas of need. Family members, care providers, and health-care professionals involved with the immediate and long-term care of individuals with PWS should be made aware of these risk factors and causes of death to improve the longevity and quality of life of these individuals (at all ages) and their family members.

\section{SUPPLEMENTARY MATERIAL}

Supplementary material is linked to the online version of the paper at http://www.nature.com/gim

\section{ACKNOWLEDGMENTS}

We acknowledge the support of the Prader-Willi Syndrome Association (USA) and families as well as the National Institute of Child Health and Human Development (grant HD02528). Partial funding was also received through an unrestricted grant from Zafgen, Inc.

\section{DISCLOSURE}

The authors declare no conflict of interest. 


\section{REFERENCES}

1. Butler MG. Prader-Willi syndrome: current understanding of cause and diagnosis. Am J Med Genet 1990;35:319-332.

2. Butler MG, Lee PDK, Whitman BY (eds). Management of Prader-Willi Syndrome, 3rd edn. Springer: New York, 2006.

3. Cassidy SB, Schwartz S, Miller JL, Driscoll DJ. Prader-Willi syndrome. Genet Med 2012:14:10-26.

4. Angulo MA, Butler MG, Cataletto ME. Prader-Willi syndrome: a review of clinical, genetic, and endocrine findings. J Endocrinol Invest 2015; 38 : 1249-1263.

5. Butler MG. Single gene and syndromic causes of obesity: Illustrative examples. Prog Mol Biol Trans/ Sci 2016;140:1-45.

6. Hill JO, Kaler M, Spetalnick B, Reed G, Butler MG. Resting metabolic rate in Prader-Willi syndrome. Dysmorphol Clin Genet 1990;4:27-32.

7. Hoybye C (ed). Prader-Will Syndrome. Nova Science Publishers: New York, 2013.

8. Butler JV, Whittington JE, Holland AJ, Boer H, Clarke D, Webb T. Prevalence of and risk factors for, physical ill-health in people with Prader-Willi syndrome: a population-based study. Dev Med Child Neurol 2002:44:248-255.

9. Hertz G, Cataletto M, Feinsilver SH, Angulo M. Sleep and breathing patterns in patients with Prader Willi syndrome (PWS): effects of age and gender. Sleep 1993;16:366-371.

10. Bittel DC, Butler MG. Prader-Willi syndrome: clinical genetics, cytogenetics and molecular biology. Expert Rev Mol Med 2005;7:1-20.

11. Butler MG. Prader-Willi syndrome: obesity due to genomic imprinting. Curr Genomics 2011;12:204-15.

12. Kim SJ, Miller JL, Kuipers PJ, et al. Unique and atypical deletions in PraderWilli syndrome reveal distinct phenotypes. Eur J Hum Genet 2011;20: 283-290.

13. Butler MG, Bittel DC, Kibiryeva N, Talebizadeh Z, Thompson T. Behavioral differences among subjects with Prader-Willi syndrome and type I or type II deletion and maternal disomy. Pediatrics 2004;113(3 Pt 1):565-573.

14. Zarcone J, Napolitano D, Peterson C, et al. The relationship between compulsive behaviour and academic achievement across the three genetic subtypes of Prader-Willi syndrome. J Intellect Disabil Res 2007; 51(Pt. 6):478-487.

15. Hartley SL, Maclean WE Jr, Butler MG, Zarcone J, Thompson T. Maladaptive behaviors and risk factors among the genetic subtypes of Prader-Willi syndrome. Am J Med Genet A 2005; 136:140-145.

16. Butler MG, Nelson TA, Driscoll DJ, Manzardo AM. Evaluation of plasma substance $P$ and beta-endorphin levels in children with Prader-Willi syndrome $J$ Rare Disord 2015; 3:1-9.

17. Swaab DF. Prader-Willi syndrome and the hypothalamus. Acta Paediatr Supp/ 1997:423:50-54

18. DiMario FJ Jr, Burleson JA. Cutaneous blood flow and thermoregulation in Prader-Willi syndrome patients. Pediatr Neurol 2002;26:130-133.

19. Hayashi M, Itoh M, Kabasawa Y, Hayashi H, Satoh J, Morimatsu Y. A neuropathological study of a case of the Prader-Willi syndrome with an interstitial deletion of the proximal long arm of chromosome 15. Brain Dev 1992;14:58-62.
20. Schrander-Stumpel C, Sijstermans H, Curfs L, Fryns JP. Sudden death in children with Prader-Willy syndrome: a call for collaboration. Genet Couns 1998;9:231-2.

21. Muralidhar B, Butler MG. Methylation PCR analysis of Prader-Willi syndrome, Angelman syndrome, and control subjects. Am J Med Genet 1998;80:263-265.

22. Kubota T, Sutcliffe JS, Aradhya S, et al. Validation studies of SNRPN methylation as a diagnostic test for Prader-Willi syndrome. Am J Med Genet 1966;66:77-80.

23. Lionti T, Reid SM, Rowell MM. Prader-Willi syndrome in Victoria: mortality and causes of death. J Paediatr Child Health 2012; 48:506-511.

24. Schrander-Stumpel CT, Curfs LM, Sastrowijoto P, Cassidy SB, Schrander JJ, Fryns JP. Prader-Willi syndrome: causes of death in an international series of 27 cases. Am J Med Genet 2004;A.124A:333-338.

25. Van Vliet G, Deal CL, Crock PA, Robitaille Y, Oligny LL. Sudden death in growth hormone-treated children with Prader-Willi syndrome. J Pediatr 2004;144 129-131.

26. Eiholzer U. Deaths in children with Prader-Willi syndrome. Horm Res 2005:63:33-39.

27. Stevenson DA, Heinemann J, Angulo M, et al. Deaths due to choking in PraderWilli syndrome. Am J Med Genet 2007;A. 143A:484-487.

28. Stevenson DA, Heinemann J, Angulo M, et al. Gastric rupture and necrosis in Prader-Willi syndrome. J Pediatr Gastroenterol Nutr 2007:45:272-274.

29. Nagai T, Obata K, Tonoki H, et al. Cause of sudden, unexpected death of PraderWilli syndrome patients with or without growth hormone treatment. Am J Med Genet 2005;136A:45-48

30. Tauber M, Diene G, Molinas $C$, Hébert M. Review of 64 cases of death in children with Prader-Willi syndrome (PWS). Am J Med Genet 2008;A.14 6A:881-887.

31. Whittington JE, Holland AJ, Webb T, Butler J, Clarke D, Boer H. Population prevalence and estimated birth incidence and mortality rate for people with Prader-Willi syndrome in one UK Health Region. J Med Genet 2001;38:792-798.

32. Butler MG, Lee J, Cox DM, et al. Growth charts for Prader-Willi syndrome during growth hormone treatment. Clin Pediatr (Phila) 2016;55:957-974.

33. Ledbetter DH, Riccardi VM, Airhart SD, Strobel RJ, Keenan BS, Crawford JD. Deletions of chromosome 15 as a cause of the Prader-Willi syndrome. N Engl J Med 1981:304:325-329.

34. Butler MG, Palmer CG. Parental origin of chromosome 15 deletion in PraderWilli syndrome. Lancet 1983:1:1285-1286.

35. Nicholls RD, Knoll JH, Butler MG, Karam S, Lalande M. Genetic imprinting suggested by maternal heterodisomy in nondeletion Prader-Willi syndrome. Nature 1989;342:281-285

36. Kuwano A, Mutirangura A, Dittrich B, et al. Molecular dissection of the PraderWilli/Angelman syndrome region (15q11-13) by YAC cloning and FISH analysis. Hum Mol Genet 1992;1:417-425.

37. Driscoll DJ, Waters MF, Williams CA, et al. DNA methylation imprint, determined by the sex of the parent, distinguishes the Angelman and Prader-Willi syndromes. Genomics 1992;13:917-924.

38. Wang NJ, Liu D, Parokonny AS, Schanen NC. High-resolution molecular characterization of 15q11-q13 rearrangements by array comparative genomic hybridization (array CGH) with detection of gene dosage. Am J Hum Genet 2004;75:267-281. 